\title{
APPLICATION OF THE COUPLING DETECTION TO THE ANALYSIS OF THE LOW-FREQUENCY RHYTHMS IN THE AUTONOMIC CONTROL OF CIRCULATION
}

\author{
Anatoly Karavaev \\ Laboratory of Nonlinear Dynamics \\ Modelling \\ Saratov Branch of the Institute \\ of RadioEngineering and Electronics \\ of Russian Academy of Sciences. \\ Department of Innovative Cardiological \\ Information Technology, \\ Institute of Cardiological Research \\ Saratov State Medical University. \\ Department of Dynamic Modeling \\ and Biomedical Engineering \\ Saratov State University. \\ Russia \\ karavaevas@gmail.com \\ Yurii Ishbulatov \\ Department of Innovative Cardiological \\ Information Technology, \\ Institute of Cardiological Research \\ Saratov State Medical University. \\ Department of Dynamic Modeling \\ and Biomedical Engineering \\ Saratov State University. \\ Russia \\ ishbulatov95@mail.ru
}

\author{
Viktoria Skazkina \\ Laboratory of Nonlinear Dynamics \\ Modelling \\ Saratov Branch of the Institute \\ of RadioEngineering and Electronics \\ of Russian Academy of Sciences. \\ Department of Dynamic Modeling \\ and Biomedical Engineering \\ Saratov State University. \\ Russia \\ skazkinavv@yandex.ru
}

Article history:

Received 14.10.2019, Accepted 26.11.2019

\begin{abstract}
Many authors emphasize the fundamental and practical importance of investigation of the low-frequency rhythm in the brain and cardiac signals that correlate with the activity of the autonomic control of circulation. However complexity, nonstationarity and high nonlinearity of the system complicate the research and require application of the specialized methods. This study aims to compare the methods of coupling detection that were adapted to the detection of coupling between the loops of autonomic control of circulation. The analysis of the data from healthy subject demonstrated that the methods based on the analysis of phase dynamics and coherence function calculations are adequate for the investigation of coupling patterns in the autonomic control of circulation. However the results of both methods require the control of statistical significance.
\end{abstract}

\section{Key words}

Coupling, autonomic control, nonlinear dynamics, electroencephalogram, cardiovascular system, respiration

\section{Introduction}

Understanding of the coupling between the infra-slow $0.1 \mathrm{~Hz}$ rhythms in electroencephalogram (EEG) and the activity of autonomic control of circulation is important from the fundamental understanding and can be applied in medical diagnostics [Aladjalova, 1957; Vandenhouten et al., 2000].

Recently we studied the synchronization of the infraslow oscillations of the EEG [Karavaev et al., 2018; Karavaev et al., 2016] with respiration and the activity of 

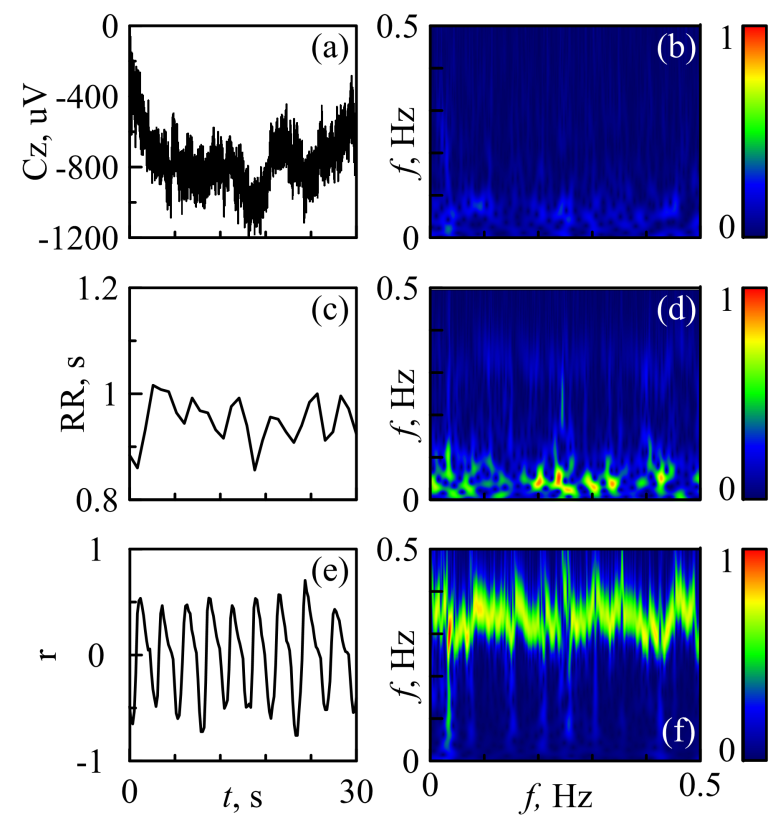

Figure 1. Time series of (a) - Cz EEG lead, (c) - RR and (e) - respiration (in nominal units). Normalized wavelet-spectra for the (b) $-\mathrm{Cz}$ EEG lead, (d) - RR and (f) - the respiratory signal.

autonomic control of circulation [Ponomarenko et al., 2013] in awake healthy subjects during the experiments with active respiration.

The subjects were instructed to inhale on an audio stimulus which frequency was changing according to the preset law.

To reveal the coupling patterns in the experimental data we used the original method based on the phase dynamic analysis. In the several stages of the experiment we detected the phase and frequency synchronization of the infra-slow EEG oscillations with respiration and revealed the self-exciting brain structures responsible for the generations of the infra-slow EEG oscillations.

In [Karavaev et al., 2018] the estimation of the coherence function was also applied to the same data. The next step is to study the patterns of directional coupling of respiration with the infra-slow EEG oscillations and the activity of autonomic control of circulation.

The aim of this study is to investigate the applicability of the nonlinear method based on phase dynamic modeling to detection of the coupling of respiration with the slow $0.1 \mathrm{~Hz}$ oscillations in EEG potentials and the sequence of RR-intervals (RR) in resting awake healthy subjects that breathe spontaneously.

\section{Experimental Data}

The 30-minutes non-invasive scalp EEG, I-standard lead electrocardiograms (ECG) and the signals of respiration were simultaneously recorded from three subjects that were breathing spontaneously.

The standard "10-20" protocol was used for registration of the non-invasive 19- lead EEG and the abdominal strain gauge sensor was used to record respiration.

The signals were recorded with the standard certified device Encephalan-EEGR-19/26 (Medicom MTD, Russia) at 16 bits resolution and 250 samples per second. The bandwidth for the EEG channels was $0.0016-80 \mathrm{~Hz}$, $0.003-80 \mathrm{~Hz}$ for the ECG channel and $0.003-80 \mathrm{~Hz}$ for the signal of respiration.

\section{Methods}

We compared three methods of coupling detection.

The first method we used was estimation of the phase coherence $\rho$ [Mormann et al., 2000]. It can be calculated from the phases difference $\Delta \phi\left(t_{n}\right)=\phi_{x}\left(t_{n}\right)-\phi_{y}\left(t_{n}\right)$ of two oscillations $X$ and $Y$ as:

$$
\rho=\left|\left\langle\exp \left(i \Delta \phi\left(t_{n}\right)\right)\right\rangle\right|
$$

where $i$ - imaginary unit, $t_{n}=n \Delta t$ - discrete time, $\Delta t$ - sample time, $n$ - sample number, $\langle\ldots\rangle$ is the time averaging. Index $\rho$ can reach the value of 1 when the oscillations are totally synchronized and the phase difference is constant.

Index $\rho$ reaches 0 when two oscillations are not coupled. The values in between 0 and 1 correspond with weak coupling.

The second approach we used was estimation of coherence function $C(f)$ that reveals the coupling between individual spectral components of the two systems and is based on the analysis of the cross-spectra [White et al., 1990]. For two oscillations $X$ and $Y$ it can be calculated as:

$$
C_{x y}(f)=\frac{S_{x y}(f)}{\sqrt{\left|S_{x}(f)\right|\left|S_{y}(f)\right|}},
$$

where $S_{x}(f)$ and $S_{y}(f)$ - are $X$ and $Y$ power spectra, $\left(\left|S_{x}(f)\right|>0,\left|S_{y}(f)\right|>0\right), S_{x y}(f)$ - cross spectrum. $C_{x y}(f)$ can reach 1 if the phases and the amplitudes are matching. $C_{x y}(f)=0$ if they do not match. $C_{x y}(f)$ in between 0 and 1 suggest weak coupling between the amplitudes and the phases of the systems.

The statistical significance of the phase coherence and the coherence function were controlled with the amplitude adjusted Fourier transform (AAFT) surrogate data [Schreiber et al., 1996] that was generated by randomizing the phases of the signal's Fourier-harmonics.

The third method we used was developed to detect presence and direction of the coupling and is based on the phase dynamics modeling [Rosenblum et al., 2001]. We used the time-series to construct the individual predictive models:

$$
\begin{array}{r}
\phi_{x}\left(t_{n}+\Delta\right)-\phi_{x}\left(t_{n}\right)= \\
F_{x}\left[\phi_{x}\left(t_{n}\right), \phi_{y}\left(t_{n}-\tau\right), \vec{a}_{x}\right],
\end{array}
$$



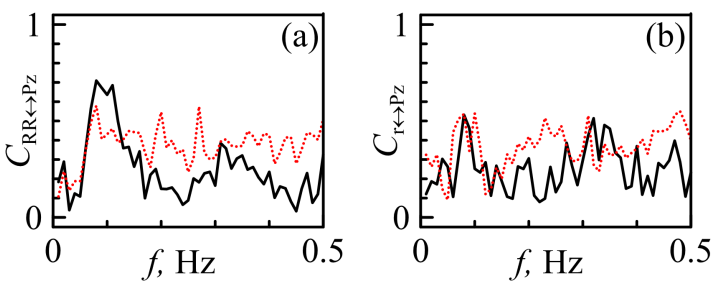

Figure 2. The coherence function of the lead Pz EEG and (a) RR, (b) - respiration for one of the subjects. Solid line is the value of the coherence function. Dotted line is $95 \%$ significance level.

where $\Delta$ is a time-lag between the time-series, $\tau$ is a time lag and $\vec{a}_{x}$ is a vector with coefficients of 3rd order trigonometric polynomial. To detect the coupling we estimated the strengths with which the second system affects the first:

$$
G_{x y}=\frac{1}{2 \pi^{2}} \iint_{0}^{2 \pi} \frac{\delta F_{x}}{\delta \phi_{x}} d \phi_{x} d \phi_{y}
$$

$G_{y x}$ was calculated in the same manner. The statistical significance of this method results was controlled with the analytical approach described in details in [Smirnov et al., 2003] at the significance level $p=0.05$.

\section{Results}

The typical experimental signals and their wavelet spectra are presented in the fig. 1. It is evident from the spectra that in the RR variability signals the characteristic oscillations are present in the LF and HF ranges and in the respiratory signal there are oscillations on the main frequency of the spontaneous respiration (fig.1 d,f).

However the spectral analysis of the EEG signals did not reveal any low-frequency oscillations in the LF or HF ranges (fig $1 \mathrm{~b}$ ). This observation correlates well with the existing data and can be explained by the complexity and comparatively low power of the infra-slow potentials in the EEG [Knyazev, 2016].

We used the abovementioned methods of coupling detection to reveal the coupling patterns of the respiratory signal with the infra-slow EEG oscillations and the rhythms in the RR. The coupling between the EEG potentials and the RR rhythms was analyzed in the 0.04-0.15 Hz range (so-called Low Frequency - LF range [Camm et al., 1996]). Coupling between the EEG potentials and the signal of respiration was analyzed in the $0.15-0.4 \mathrm{~Hz}$ range (High Frequency HF range [Camm et al., 1996]).

The results of the analysis based on the phase coherence calculations were not statistically significant for either the LF or the HF range. The $\rho$ index calculated between the EEG potentials in the HF band and the signal of respiration was $0.14 \pm 0.08$ (mean \pm standard deviation) as averaged from all subjects and EEG leads. The $\rho$ index index calculated between the EEG potentials in the LF band and the RR rhythms was $0.16 \pm 0.07$. The $\rho$ index between the RR rhythms in the LF range and the respiratory signals was $0.18 \pm 0.12$ and $0.16 \pm 0.02$ in the HF range.

The coherence function $C(f)$ revealed the statistically significant coupling between the $0.1 \mathrm{~Hz}$ rhythms in the RR signals and several EEG leads (Fig. 2 a). The $C(f)$ calculated between the HF EEG rhythms and respiration is lower and just above the verge of the significance level (Fig. 2 b).

For the HF rhythms in the EEG leads and the respiratory signal the $C(f)$ is $0.59 \pm 0.05$ as averaged from all subjects and EEG leads. For the LF rhythms in the EEG leads and the RR signals the $C(f)$ is $0.90 \pm 0.04$ and for the $\mathrm{HF}$ range it is $0.16 \pm 0.02$ [Karavaev et al., 2018].

The results of coherence function calculations suggest that the $0.1 \mathrm{~Hz}$ rhythms in the EEG and RR signals and $0.1 \mathrm{~Hz}$ rhythms in the EEG and respiratory signals are highly coherent. The maximal values that coherence function reached was 0.83 between the Fz EEG lead and the RR variability signals. The maximum was observed at the $0.09 \mathrm{~Hz}$ frequency. The maximal coherence between the P4 EEG lead and the respiratory signal was 0.83 at $0.25 \mathrm{~Hz}$.

The typical examples of coupling diagnostics via the phase modeling are presented in fig. 3. The panels show the dependence of the coupling index $G$ from the delay time $\tau$ The vertical whiskers represent the significance level as calculated with the analytical approach from [Smirnov et al., 2003]. The analysis revealed that the estimation of the delay time that corresponds with the maximal $G$ shows high variance. The strongest variance was observed for the central EEG leads, the coefficient of variation was $141 \%$ for the C4 EEG lead. The lowest variance was observed for the occipital leads, the coefficient of variation was $11 \%$ for the P3 EEG lead.

The fluctuations of the $\tau$ are most likely related to the nonstationarity of the experimental data, complexity of the oscillations and presence of noises of various origins. Therefore the estimation of the coupling delay is a nontrivial problem that exceeds the scope of this study. For the further analysis we took into account the maximal value of the $G(\tau)$ dependence. The approach based on the phase dynamics modeling revealed the patterns of coupling between the infra-slow EEG potentials and the RR rhythms in the LF frequency range that is mostly related to the sympathetic control of circulation [Camm et al., 1996]. Coupling in the direction from the RR signal to the EEG leads is more pronounced (Fig. 3d) than the coupling in the opposite direction (Fig. 3c). The maximal value of $G$ for the coupling in direction from the RR rhythms to the EEG lead was reached for the Pz lead and was 0.11. Maximal $G$ for the coupling in the opposite direction was for the T6 lead and was 0.07.

The influence of the respiratory signal on the infraslow EEG oscillations in the HF range is typicaly not statistically significant (Fig. 3e). For most EEG leads we 


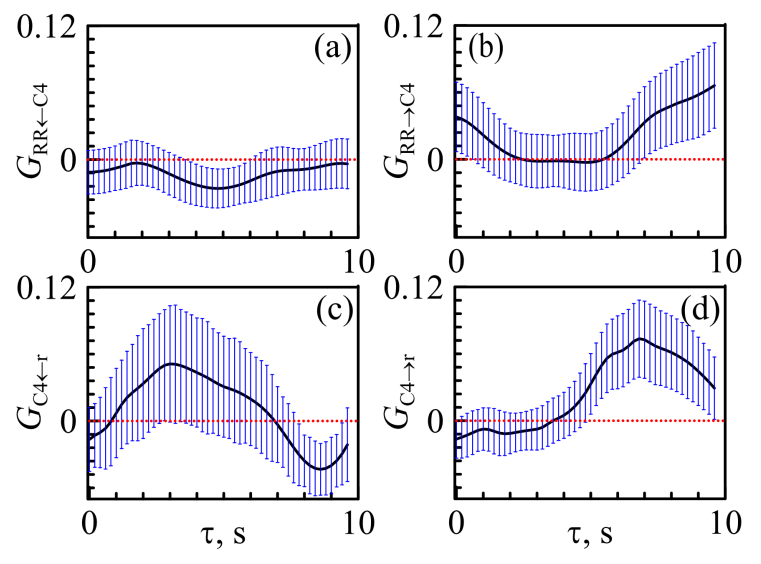

Figure 3. The directional coupling index $\mathrm{G}$ (solid line) estimated between the C4 EEG lead and RR (a, b) and respiratory signal (c, d) in one of the subjects. The $\Delta$ was set to $10 \mathrm{~s}$. The arrows in the title of the vertical axis indicate the direction of the coupling being tested. Whiskers mark the analytical $95 \%$ confidence interval for the difference between $\mathrm{G}$ and 0 . A zero $\mathrm{G}$ value is indicated by a horizontal dotted red line. Bottom axis marks a time delay between the signals.

revealed significant influence from the infra-slow EEG oscillations on the respiratory signal (Fig. 3f). The maximal value of $G$ for the coupling in direction from the respiratory rhythm to the EEG lead was reached for the T6 lead and was 0.15. Maximal $G$ for the coupling in the opposite direction was for the T5 lead and was 0.19.

\section{Conclusion}

The study demonstrated that the analysis of the coupling patterns in the autonomic control of circulation including the brain centers can be carried out with calculation of the coherence function or the method based on the phase dynamics modeling. In the case of coherence function it is necessary to control the statistical significance via AAFT surrogate data [Schreiber et al., 1996]. The analytical [Smirnov et al., 2003] approach to the control of statistical significance must be adopted in case of the phase dynamics modeling. The coupling coefficients were estimated for three subjects however the standard deviation of the estimation was too high due to the small sample size. Therefore the patterns of directional couplings need further investigation.

\section{Acknowledgements}

This work was supported by the Russian Science Foundation, Grant No.19-12-00201.

\section{References}

Aladjalova, N. A. (1957). Infra-slow rhythmic oscillations of the steady potential of the cerebral cortex. $\mathrm{Na}$ ture, 179, pp. 957-969.

Karavaev, A. S., Kiselev, A. R., Runnova, A. E., Zhuravlev, M. O., Borovkova, E. I., Prokhorov, M. D.,
Ponomarenko, V. I., Pchelintseva, S. V., Efremova, T. Yu., Koronovskii, A. A., and Hramov, A. E. (2018). Synchronization of infra-slow oscillations of brain potentials with respiration. Chaos, 28, pp. 081102.

Karavaev, A. S., Kiselev, A. R., Runnova, A. E., and Koronovskii, A. A. (2018). Coherence of low-frequency oscillations of electroencephalogram and the process of autonomous regulation of heart rhythm. 2018 2nd School on Dynamics of Complex Networks and their Application in Intellectual Robotics (DCNAIR), Saratov, Russia, Oct. 8-10, pp. 127-129.

Karavaev, A. S., Runnova, A. E., Borovkova, E. I., Ishbulatov, Y. M., Khorev, V. S., Kiselev, A. R., Zhuravlev, M. O., Ponomarenko, V. I., Prokhorov, M. D., Bezruchko, B. P., and Koronovskii, A. A. (2016). Synchronization of low-frequency rhythms in electroencephalogram by respiration with frequency linearly dependent from time. Saratov Journal of Medical Scientific Research, 12 (4), pp. 541-548.

Knyazev, G. G. (2016). EEG delta oscillations as a correlate of basic homeostatic and motivational processes. Neuroscience and Biobehavioral Reviews, 36 (1), pp. 677-695.

Mormann, F., Lehnertz, K., David, P., and Elger, C. E. (2000). Mean phase coherence as a measure for phase synchronization and its application to the EEG of epilepsy patients. Physica D, 144 (3-4), pp. 358369.

Ponomarenko, V. I., Prokhorov, M. D., Karavaev, A. S., Kiselev, A. R., Gridnev, V. I., and Bezruchko, B. P. (2013). Synchronization of low-frequency oscillations in the cardiovascular system: Application to medical diagnostics and treatment. The European Physical Journal Special Topics, 222, pp. 2687-2696.

Rosenblum, M. G., and Pikovsky, A. S. (2001). Detecting direction of coupling in interacting oscillators. Physical Review E, 64, pp. 045202.

Schreiber, T., and Schmitz, A. (1996). Improved surrogate data for nonlinearity tests. Physical Review Letters, 77 (4), pp. 635-638.

Smirnov, D., and Bezruchko, B. (2003). Estimation of interaction strength and direction from short and noisy time series. Physical Review E, 68, pp. 046209.

Camm, A. J., Marek, M., Bigger, J. T., et al. (1996). Heart rate variability. Standards of measurement, physiological interpretation, and clinical use. Task Force of the European Society of Cardiology and the North American Society of Pacing and Electrophysiology. Circulation, 17, pp. 354-381.

Vandenhouten, R., Lambertz, M., Langhorst, P., and Grebe, R. (2000). Nonstationary time-series analysis applied to investigation of brainstem system dynamics. IEEE Transactions on Biomedical Engineering, 47, pp. 729-737.

White, L. B., and Boashash, B. (1990). Cross spectral analysis of nonstationary processes. IEEE Transactions on Information Theory, 36 (4), pp. 830-835. 